\title{
Dephasing due to Intermode Coupling in Superconducting Stripline Resonators
}

\author{
Eyal Buks \\ Department of Electrical Engineering, Technion, Haifa 32000 Israel \\ Bernard Yurke \\ Bell Laboratories, Lucent Technologies, 600 Mountain Avenue, Murray Hill, NJ 07974
}

(Dated: July 13, 2018)

\begin{abstract}
The nonlinearity exhibited by the kinetic inductance of a superconducting stripline couples stripline resonator modes together in a manner suitable for quantum non-demolition measurement of the number of photons in a given resonator mode. Quantum non-demolition measurement is accomplished by coherently driving another resonator mode, referred to as the detector mode, and measuring its response. We show that the sensitivity of such a detection scheme is directly related to the dephasing rate induced by such an intermode coupling. We show that high sensitivity is expected when the detector mode is driven into the nonlinear regime and operated close to a point where critical slowing down occurs.
\end{abstract}

PACS numbers: 42.50.Gy, 42.65.Yj, 42.50.Dv

\section{INTRODUCTION}

The resistive and inductive nonlinearity exhibited by superconducting striplines and microstrips originates from the nonuniform distribution of the microwave current over the transmission line cross section. Along the edges, where the current density obtains its peak value, the current density can become overcritical, even with relatively moderate power levels. As a result, the superconducting current distribution may change as the total current within the transmission line changes. This, in turn, causes both the inductance $L$ and resistance $R$ per unit length to become current dependent [1].

Such nonlinearity introduces coupling between different modes in a stripline resonator. In the rotating wave approximation (RWA) such coupling adds a term to the Hamiltonian of the system given by $\sum_{n^{\prime} \neq n^{\prime \prime}} \hbar \lambda_{n^{\prime} n^{\prime \prime}} N_{n^{\prime}} N_{n^{\prime \prime}}$, where $N_{n}=A_{n}^{\dagger} A_{n}$ is the number operator of mode $n$, and the coupling constants $\lambda_{n^{\prime} n^{\prime \prime}}$ are given by A22 As was shown in Ref. 2], such intermode coupling may allow a non-demolition measurement [3], 4] of the number of photons in a signal mode by intensively driving another mode, called a detector mode (or pump), and monitoring its response near the pump frequency. Such a measurement scheme is characterized by a measurement time, defined as the time needed to distinguish between initial states of the signal mode having different number of photons. Single-photon detection can be realized if the measurement time can be made shorter than the lifetime of a Fock state of the signal mode.

However, according to the Bohr's complementarity principle [5], in the limit of single-photon detection, dephasing is expected to come into play, leading to broadening of the resonance line shape of the signal mode. Thus, a which-path-like experiment can be employed to test whether single-photon detection is possible. This can be done by monitoring the resonance response of the signal mode and the way it is affected by driving the de- tector mode. Observing a broadening comparable to or larger than the width of the resonance when the detector is not driven implies that single-photon detection is possible in principle, namely, the measurement time is comparable to or shorter than the lifetime of a photon in the mode.

In practice, however, as we show below, the coupling constants $\lambda_{n^{\prime} n^{\prime \prime}}$ in superconducting stripline resonators are far too weak to allow single-photon detection when the detector mode is taken to have a linear response. On the other hand, the same kinetic inductance which leads to intermode coupling also gives rise to a detector mode Kerr nonlinearity. In the RWA such nonlinearity adds the term $\sum_{n} K_{n}\left(N_{n}\right)^{2}$ to the Hamiltonian of the system, where the Kerr constants $K_{n}$ are given by A23 Such Duffing-like nonlinearity leads to bistability when the drive level exceeds some critical value. In the bistable regime of operation the response of the detector mode exhibits hysteresis and jumps as the frequency of the drive or its amplitude is varied.

In the present work we investigate the dephasing rate induced by the driven detector mode on the signal mode. We consider a detector mode having both a Kerr-like nonlinearity and nonlinear damping. We find that the dephasing rate diverges as one approaches a point where the response of the detector mode exhibits a jump. At such a point the slope of the response vs. frequency is infinite. This result suggests that strong dephasing can be induced when driving the detector mode in the nonlinear regime, even with relatively weak intermode coupling. Thus, operating such a photon detector in this regime may allow enhanced sensitivity.

The dynamics of a driven single-mode stripline resonator having a Kerr-like nonlinearity, in addition to linear and nonlinear damping 6], was studied recently by us in 7]. The equations of motion were derived using the input-output theory of Gardiner and Collett [8, 9]. We have shown that in the appropriate limit such a system can serve as a phase-sensitive amplifier whose 
noise performance exceeds that of the quantum limits imposed on linear, phase-insensitive parametric amplifiers [10]. Moreover, we have studied degradation of device performance, due to two-photon absorption originated by nonlinear resistance, which can be significant in such devices (see also 11, 12, 13, 14]).

A closely related problem, the non-demolition measurement of Fock states in mesoscopic mechanical oscillators, was recently studied theoretically in [15]. Anharmonic effects on such a measurement were further studied in [16]. In the RWA the Hamiltonian of the mechanical systems, studied in [15] and [16], and the one of a stripline resonator presented here, are similar. However, our analysis goes beyond that of [15] and [16 by taking into account the effect of nonlinear damping 17]. Moreover, while the emphasis in Refs. [15] and [16] is on the properties of the detection scheme, including a thorough consideration of the phase diffusion back-action, here we focus on the relation between decoherence and the distinguishability between states with different numbers of photons.

\section{SINGLE-PHOTON DETECTOR}

The Hamiltonian of the system is given by

$$
H=H_{s}+H_{D}+V \text {. }
$$

Here $H_{s}$ is the Hamiltonian of the mode of the photons to be detected (signal mode),

$$
H_{s}=\hbar \omega_{s} N_{s}
$$

where $N_{s}=A_{s}^{\dagger} A_{s}$ is the number operator of the signal mode. $H_{D}$ is the Hamiltonian of the detector. $V$ is the coupling between the signal mode and the detector, and is taken to have the form

$$
V=\hbar \lambda N_{s} W
$$

where $W$ is an operator on the Hilbert space of the detector.

The Heisenberg equation of motion can be used to show that the number of photons in the signal mode $N_{s}$ is a constant of motion

$$
i \hbar \frac{d}{d t}\left\langle N_{s}\right\rangle=\left\langle\left[N_{s}, H\right]\right\rangle=0
$$

Thus, the detection process in this case is a quantum non-demolition measurement of the number $N_{s}$ [2].

To study the ability of the detector to measure $N_{s}$ we follow the approach presented in Refs. [18], [19], 20]. Consider an initial state at time $t=0$, having a superposition of 0 and 1 photons

$$
|\psi(t=0)\rangle=\left(|0\rangle_{s}+|1\rangle_{s}\right) \otimes\left|\chi_{i}\right\rangle_{D},
$$

where $|n\rangle_{s}$ is a Fock state of the signal mode and $\left|\chi_{i}\right\rangle_{D}$ represents an initial state of the detector. At a later time $t>0$ the state in the Schrödinger representation will evolve into

$$
|\psi(t)\rangle=u(t)|\psi(t=0)\rangle
$$

where the time evolution operator $u(t)$ is given by

$$
u(t)=\mathcal{T} \exp \left[-\frac{i}{\hbar} \int_{0}^{t} d t^{\prime} H\left(t^{\prime}\right)\right]
$$

and $\mathcal{T}$ is the time-ordering operator. Equation 1 yields

$$
\begin{aligned}
& u(t)|n\rangle_{s} \otimes\left|\chi_{i}\right\rangle_{D} \\
& =\mathcal{T} \exp \left[-\frac{i}{\hbar} \int_{0}^{t} d t^{\prime}\left[H_{D}+\hbar\left(\omega_{s}+\lambda W\right) N_{s}\right]\right]|n\rangle_{s} \otimes\left|\chi_{i}\right\rangle_{D} \\
& =|n\rangle_{s} \otimes u_{n}\left|\chi_{i}\right\rangle_{D},
\end{aligned}
$$

where

$$
u_{n}=e^{-i n \omega_{s} t} \mathcal{T} \exp \left[-\frac{i}{\hbar} \int_{0}^{t} d t^{\prime}\left(H_{D}+n \hbar \lambda W\right)\right] .
$$

Thus, as expected, the time evolution preserves $N_{s}$, and

$$
|\psi(t)\rangle=|0\rangle_{s} \otimes\left|\chi_{0}\right\rangle_{D}+|1\rangle_{s} \otimes\left|\chi_{1}\right\rangle_{D}
$$

where $\left|\chi_{0}\right\rangle_{D}=u_{0}\left|\chi_{i}\right\rangle_{D}$ and $\left|\chi_{1}\right\rangle_{D}=u_{1}\left|\chi_{i}\right\rangle_{D}$. The distinguishability between 0 and 1 photons associated with this state is characterized by the parameter

$$
\nu \equiv\left|\left\langle A_{s}\right\rangle\right|^{2}=\left.\left.\right|_{D}\left\langle\chi_{0} \mid \chi_{1}\right\rangle_{D}\right|^{2}=\left.{ }_{D}\left\langle\chi_{i}\left|u_{0}^{\dagger} u_{1}\right| \chi_{i}\right\rangle_{D}\right|^{2}
$$

Our goal is to find the characteristic time scale $\tau_{\varphi}$ over which $\nu$ decays from its initial value $\nu=1$ at time $t=0$. Regarding the coupling parameter $\lambda$ as small, one can evaluate $\nu$ using perturbation theory. To lowest order in $\lambda$ one finds using B10

$$
\nu=1-\lambda^{2} \int_{0}^{t} d t^{\prime} \int_{0}^{t} d t^{\prime \prime} K\left(t^{\prime}, t^{\prime \prime}\right)
$$

where

$$
\hat{K}\left(t^{\prime}, t^{\prime \prime}\right)=\frac{1}{2}\left[\left\langle\tilde{W}\left(t^{\prime}\right) \tilde{W}\left(t^{\prime \prime}\right)\right\rangle+\left\langle\tilde{W}\left(t^{\prime \prime}\right) \tilde{W}\left(t^{\prime}\right)\right\rangle\right],
$$

and

$$
\tilde{W}(t)=W(t)-\langle W(t)\rangle
$$

For a steady state the correlation function $\hat{K}\left(t^{\prime}, t^{\prime \prime}\right)$ is a function of $\tau=\left|t^{\prime}-t^{\prime \prime}\right|$. Moreover, it decays on some characteristic time scale $\tau_{\varphi}$, which can be identified from Eq. 12 [19]

$$
\frac{1}{\tau_{\varphi}}=\lambda^{2} \int_{-\infty}^{\infty} d \tau K(\tau)
$$

where $K(\tau)=\hat{K}(0, \tau)$. 


\section{THE DETECTOR MODE}

The detector mode is a resonator mode distinct from the signal mode. Because it is driven to large amplitude, the Kerr nonlinearity and the linear and nonlinear damping of the mode are taken into account in our analysis. Here we review the main results of Ref. [7].

\section{A. The Hamiltonian}

The Hamiltonian is given by

$H_{D}=H_{A}+H_{K}+H_{a_{1}}+H_{a_{2}}+H_{a_{3}}+H_{T_{1}}+H_{T_{2}}+H_{T_{3}}$, where $H_{A}$ is the Hamiltonian for the detector mode

$$
H_{A}=\hbar \omega_{0} A^{\dagger} A
$$

$H_{K}$ is the Hamiltonian for the Kerr nonlinearity

$$
H_{K}=\frac{\hbar}{2} K A^{\dagger} A^{\dagger} A A
$$

$H_{a 1}, H_{a 2}$ and $H_{a 3}$ are the Hamiltonians for the bath modes associated with the dissipative elements

$$
\begin{aligned}
& H_{a 1}=\int d \omega \hbar \omega a_{1}^{\dagger}(\omega) a_{1}(\omega), \\
& H_{a 2}=\int d \omega \hbar \omega a_{2}^{\dagger}(\omega) a_{2}(\omega), \\
& H_{a 3}=\int d \omega \hbar \omega a_{3}^{\dagger}(\omega) a_{3}(\omega) .
\end{aligned}
$$

$H_{T_{1}}$ and $H_{T_{2}}$ are the Hamiltonians for the linear dissipative elements, one of which is the port through which signals enter and leave the oscillator. These Hamiltonians linearly couple the bath modes, $a_{1}$ and $a_{2}$ respectively, to the oscillator mode $A$

$$
\begin{aligned}
& H_{T_{1}}=\hbar \int d \omega\left[T_{1} A^{\dagger} a_{1}(\omega)+T_{1}^{*} a_{1}^{\dagger}(\omega) A\right], \\
& H_{T_{2}}=\hbar \int d \omega\left[T_{2} A^{\dagger} a_{2}(\omega)+T_{2}^{*} a_{2}^{\dagger}(\omega) A\right] .
\end{aligned}
$$

The two-photon absorptive coupling of the resonator mode to the bath modes $a_{3}$ is modeled by a hopping Hamiltonian in which two cavity photons are destroyed for every bath photon created [21, 22, 23, 24, 25]

$$
H_{T_{3}}=\hbar \int d \omega\left[T_{3} A^{\dagger} A^{\dagger} a_{3}(\omega)+T_{3}^{*} a_{3}^{\dagger}(\omega) A A\right] .
$$

The modes are boson modes, satisfying the usual Bose commutation relations.

\section{B. Equations of motion for $A$}

The equation of motion for $A$ is given by

$$
\begin{aligned}
\frac{d A}{d t} & =-i \omega_{0} A-i K A^{\dagger} A A-\gamma A-\gamma_{3} A^{\dagger} A A \\
& -i \sqrt{2 \gamma_{1}} e^{i \phi_{1}} a_{1}^{i n}(t)-i \sqrt{2 \gamma_{2}} e^{i \phi_{2}} a_{2}^{i n}(t) \\
& -i 2 \sqrt{\gamma_{3}} e^{i \phi_{3}} A^{\dagger} a_{3}^{i n}(t),
\end{aligned}
$$

where $\gamma=\gamma_{1}+\gamma_{2}$. The damping constants are written as

$$
T_{n}=\sqrt{\frac{\gamma_{n}}{\pi}} e^{i \phi_{n}}
$$

where $\gamma_{n}$ and $\phi_{n}(n=1,2,3)$ are real, and

$$
a_{n}^{i n}(t)=\frac{1}{\sqrt{2 \pi}} \int d \omega e^{-i \omega\left(t-t_{0}\right)} a_{n}\left(t_{0}, \omega\right) .
$$

The following commutation relations exist for the bath modes

$$
\begin{aligned}
& {\left[a_{n}\left(t_{0}, \omega\right), a_{m}^{\dagger}\left(t_{0}, \omega^{\prime}\right)\right]=\delta\left(\omega-\omega^{\prime}\right) \delta_{n m},} \\
& {\left[a_{n}\left(t_{0}, \omega\right), a_{m}\left(t_{0}, \omega^{\prime}\right)\right]=0,}
\end{aligned}
$$

where $n, m=1,2,3$.

\section{Mean-Field Solution}

Since the detector mode is driven to large amplitude, compared with quantum fluctuations, the behavior of the mean-field is the classical behavior of the mode, except very near instability points. Hence, to obtain expressions for the mean-field behavior, in this section, we treat $a_{1}^{i n}$, $a_{2}^{i n}$, and $a_{3}^{i n}$ as complex numbers rather than as operators. We take the bath mode amplitudes $a_{2}^{i n}$ and $a_{3}^{i n}$ to be those entering the cavity from losses. Consequently, we take $a_{2}^{i n}=0$ and $a_{3}^{i n}=0$. The amplitude $a_{1}^{i n}$ is chosen to be that of the incoming drive and is taken to have the oscillatory time dependence

$$
a_{1}^{i n}=b_{1}^{i n} e^{-i\left(\omega_{p} t+\psi_{1}\right)},
$$

where $b_{1}^{i n}$ is a real constant. Writing $A$ as

$$
A=B e^{-i\left(\omega_{p} t+\phi_{B}\right)},
$$

where $B$ is a positive real constant, the equations of motion become

$\left[i\left(\omega_{0}-\omega_{p}\right)+\gamma\right] B+\left(i K+\gamma_{3}\right) B^{3}=-i \sqrt{2 \gamma_{1}} b_{1}^{i n} e^{i\left(\phi_{1}+\phi_{B}-\psi_{1}\right)}$.

Multiplying each side of the nonlinear equation by its complex conjugate, one obtains [26], 27]

$$
\begin{gathered}
B^{6}+\frac{2\left[\left(\omega_{0}-\omega_{p}\right) K+\gamma \gamma_{3}\right]}{K^{2}+\gamma_{3}^{2}} B^{4} \\
+\frac{\left(\omega_{0}-\omega_{p}\right)^{2}+\gamma^{2}}{K^{2}+\gamma_{3}^{2}} B^{2}-\frac{2 \gamma_{1}}{K^{2}+\gamma_{3}^{2}}\left(b_{1}^{i n}\right)^{2}=0 .
\end{gathered}
$$


Once $B^{2}$ has been determined from the above cubic algebraic equation, the phase can be found from

$$
\cot \left(\phi_{1}+\phi_{B}-\psi_{1}-\pi / 2\right)=\frac{\gamma+\gamma_{3} B^{2}}{\omega_{0}-\omega_{p}+K B^{2}}
$$

Taking the derivative of Eq. 33] with respect to $\omega_{p}$, one finds

$$
\begin{aligned}
& \frac{\partial B^{2}}{\partial \omega_{p}} \\
& =\frac{2\left(\omega_{0}-\omega_{p}+K B^{2}\right) B^{2}}{\left(\omega_{0}-\omega_{p}+2 K B^{2}\right)^{2}+\left(\gamma+2 \gamma_{3} B^{2}\right)^{2}-\left(K^{2}+\gamma_{3}^{2}\right) B^{4}} .
\end{aligned}
$$

\section{Onset of bistability point}

At the onset of bistability point the following holds

$$
\frac{\partial \omega_{p}}{\partial B^{2}}=\frac{\partial^{2} \omega_{p}}{\partial\left(B^{2}\right)^{2}}=0
$$

Such a point occurs only if the nonlinear damping is sufficiently small

$$
|K|>\sqrt{3} \gamma_{3}
$$

At this critical point the following holds

$$
\begin{gathered}
B_{c}^{2}=\frac{2 \gamma}{\sqrt{3}\left(|K|-\sqrt{3} \gamma_{3}\right)}, \\
\omega_{0}-\omega_{p c}=-\gamma \frac{K}{|K|}\left[\frac{4 \gamma_{3}|K|+\sqrt{3}\left(K^{2}+\gamma_{3}^{2}\right)}{K^{2}-3 \gamma_{3}^{2}}\right], \\
\left(b_{1 c}^{\text {in }}\right)^{2}=\frac{4}{3 \sqrt{3}} \frac{\gamma^{3}\left(K^{2}+\gamma_{3}^{2}\right)}{\gamma_{1}\left(|K|-\sqrt{3} \gamma_{3}\right)^{3}} .
\end{gathered}
$$

\section{E. Quantum fluctuations about the mean-field solution}

To determine the quantum fluctuations about the mean-field solution we write

$$
\begin{gathered}
a_{1}^{i n}=b_{1}^{i n} e^{-i\left(\omega_{p} t+\psi_{1}\right)}+c_{1}^{i n} e^{-i \omega_{p} t}, \\
a_{2}^{i n}=c_{2}^{i n} e^{-i \omega_{p} t}, \\
a_{3}^{i n}=c_{3}^{i n} e^{-i \omega_{p} t}
\end{gathered}
$$

and

$$
A=B e^{-i\left(\omega_{p} t+\phi_{B}\right)}+a e^{-i \omega_{p} t},
$$

where $B$ constitutes the mean-field amplitude of the detector mode in response to the classical drive $b_{1}^{i n}$. The operators $c_{1}^{i n}, c_{2}^{i n}, c_{3}^{i n}$, and $a$ are regarded as small and will be kept only up to linear order. In this approximation the operator $a$ satisfies the following second-order equation of motion

$$
\frac{d^{2} a}{d t^{2}}+2 \Re(w) \frac{d a}{d t}+\left(|w|^{2}-|v|^{2}\right) a=\Gamma(t),
$$

where

$$
w=i\left(\omega_{0}-\omega_{p}\right)+\gamma+2\left(i K+\gamma_{3}\right) B^{2},
$$

$$
v=\left(i K+\gamma_{3}\right) B^{2} e^{-2 i \phi_{B}},
$$

$$
\Gamma(t)=\frac{d F(t)}{d t}+w^{*} F(t)-v F^{\dagger}(t)
$$

and

$$
\begin{aligned}
F(t) & =-i \sqrt{2 \gamma_{1}} e^{i \phi_{1}} c_{1}^{i n}-i \sqrt{2 \gamma_{2}} e^{i \phi_{2}} c_{2}^{i n} \\
& -i 2 \sqrt{\gamma_{3}} B e^{i\left(\omega_{p} t+\phi_{B}+\phi_{3}\right)} c_{3}^{i n} .
\end{aligned}
$$

The solution to Eq. 45 is given by

$$
a(t)=\int_{-\infty}^{\infty} d \tau G(t-\tau) \Gamma(\tau)
$$

where

$$
G(t)=u(t) \frac{e^{-\lambda_{0} t}-e^{-\lambda_{1} t}}{\lambda_{0}-\lambda_{1}},
$$

$u(t)$ is the step function, and the eigenvalues $\lambda_{0}$ and $\lambda_{1}$ satisfy

$$
\lambda_{0}+\lambda_{1}=2 \Re(w),
$$

$$
\lambda_{0} \lambda_{1}=|w|^{2}-|v|^{2}
$$

From Eq. 35 one finds that the slope of the response function $B^{2}$ vs. $\omega_{p}$ is infinite when

$$
|w|^{2}-|v|^{2}=0
$$

Thus, using Eq. 53 one finds that at these points at least one of the eigenvalues $\lambda_{0}$ and $\lambda_{1}$ vanishes. The system eigenmode corresponding to the eigenvalue that vanishes is the one that experiences critical slowing down.

\section{F. Thermal noise}

Assuming the bath modes are in thermal equilibrium, the following hold

$$
\left\langle c_{n}^{i n}(\tau)\right\rangle=\left\langle c_{n}^{i n \dagger}(\tau)\right\rangle=0,
$$




$$
\begin{array}{r}
\left\langle c_{n}^{i n}(\tau) c_{m}^{i n}\left(\tau^{\prime}\right)\right\rangle=\left\langle c_{n}^{i n \dagger}(\tau) c_{m}^{i n \dagger}\left(\tau^{\prime}\right)\right\rangle=0, \\
\left\langle c_{n}^{i n \dagger}(\tau) c_{m}^{i n}\left(\tau^{\prime}\right)\right\rangle=\delta\left(\tau-\tau^{\prime}\right) \delta_{n m}\left\langle n_{\omega_{0}}\right\rangle, \\
\left\langle c_{n}^{i n}(\tau) c_{m}^{i n \dagger}\left(\tau^{\prime}\right)\right\rangle=\delta\left(\tau-\tau^{\prime}\right) \delta_{n m}\left(\left\langle n_{\omega_{0}}\right\rangle+1\right),
\end{array}
$$

where

$$
\left\langle n_{\omega}\right\rangle=\frac{1}{e^{\beta \hbar \omega}-1},
$$

$n, m=1,2,3$ and $\beta=1 / k_{B} T$.

\section{THE DEPHASING RATE}

Since the interaction Hamiltonian between modes is bilinear in the number operators for the modes, one has $W=A^{\dagger} A$, or using Eq. 44

$$
W(t)=B^{2}+B\left[a(t) e^{i \phi_{B}}+a^{\dagger}(t) e^{-i \phi_{B}}\right]+a^{\dagger}(t) a(t) .
$$

Using Eqs. [50] and 55] one finds

$$
\langle a(t)\rangle=\left\langle a^{\dagger}(t)\right\rangle=0 .
$$

Moreover, keeping terms only up to first order in $a$, one finds

$$
\tilde{W}(t)=W(t)-\langle W(t)\rangle=B\left[a(t) e^{i \phi_{B}}+a^{\dagger}(t) e^{-i \phi_{B}}\right] .
$$

In order to use Eq. 15 to calculate the dephasing rate, some expectation values must be evaluated. Using Eqs. 55] 56] 57] and 58, one finds

$$
\left\langle F(\tau) F\left(\tau^{\prime}\right)\right\rangle=0,
$$

$$
\left\langle F^{\dagger}(\tau) F^{\dagger}\left(\tau^{\prime}\right)\right\rangle=0
$$

$$
\left\langle F(\tau) F^{\dagger}\left(\tau^{\prime}\right)\right\rangle=\left(\lambda_{0}+\lambda_{1}\right) \delta\left(\tau-\tau^{\prime}\right)\left\langle n_{\omega_{0}}\right\rangle
$$

and

$$
\left\langle F^{\dagger}(\tau) F\left(\tau^{\prime}\right)\right\rangle=\left(\lambda_{0}+\lambda_{1}\right) \delta\left(\tau-\tau^{\prime}\right)\left(\left\langle n_{\omega_{0}}\right\rangle+1\right) .
$$

Thus, using Eq. [50] one finds

$$
\begin{aligned}
& \left\langle a(t) a\left(t^{\prime}\right)\right\rangle \\
& =\int_{-\infty}^{\infty} d \tau \int_{-\infty}^{\infty} d \tau^{\prime} G(t-\tau) G\left(t^{\prime}-\tau^{\prime}\right)\left\langle\Gamma(\tau) \Gamma\left(\tau^{\prime}\right)\right\rangle \\
& =\left(\lambda_{0}+\lambda_{1}\right)\left\langle n_{\omega_{0}}\right\rangle v \int_{-\infty}^{\infty} d \tau \frac{d G(t-\tau)}{d \tau} G\left(t^{\prime}-\tau\right) \\
& +\left(\lambda_{0}+\lambda_{1}\right)\left(\left\langle n_{\omega_{0}}\right\rangle+1\right) v \int_{-\infty}^{\infty} d \tau G(t-\tau) \frac{d G\left(t^{\prime}-\tau\right)}{d \tau} \\
& -\left(\lambda_{0}+\lambda_{1}\right)\left(2\left\langle n_{\omega_{0}}\right\rangle+1\right) v w^{*} \int_{-\infty}^{\infty} d \tau G(t-\tau) G\left(t^{\prime}-\tau\right)
\end{aligned}
$$

and

$$
\begin{aligned}
& \left\langle a^{\dagger}(t) a\left(t^{\prime}\right)\right\rangle \\
& =\int_{-\infty}^{\infty} d \tau \int_{-\infty}^{\infty} d \tau^{\prime} G(t-\tau) G\left(t^{\prime}-\tau^{\prime}\right)\left\langle\Gamma^{\dagger}(\tau) \Gamma\left(\tau^{\prime}\right)\right\rangle \\
& =\left(\lambda_{0}+\lambda_{1}\right)\left(\left\langle n_{\omega_{0}}\right\rangle+1\right) \int_{-\infty}^{\infty} d \tau \frac{d G(t-\tau)}{d \tau} \frac{d G\left(t^{\prime}-\tau\right)}{d \tau} \\
& -\left(\lambda_{0}+\lambda_{1}\right)\left(\left\langle n_{\omega_{0}}\right\rangle+1\right) w^{*} \int_{-\infty}^{\infty} d \tau \frac{d G(t-\tau)}{d \tau} G\left(t^{\prime}-\tau\right) \\
& -\left(\lambda_{0}+\lambda_{1}\right)\left(\left\langle n_{\omega_{0}}\right\rangle+1\right) w \int_{-\infty}^{\infty} d \tau G(t-\tau) \frac{d G\left(t^{\prime}-\tau\right)}{d \tau} \\
& +\left(\lambda_{0}+\lambda_{1}\right)\left(\left\langle n_{\omega_{0}}\right\rangle+1\right)|w|^{2} \int_{-\infty}^{\infty} d \tau G(t-\tau) G\left(t^{\prime}-\tau\right) \\
& +\left(\lambda_{0}+\lambda_{1}\right)\left\langle n_{\omega_{0}}\right\rangle|v|^{2} \int_{-\infty}^{\infty} d \tau G(t-\tau) G\left(t^{\prime}-\tau\right) .
\end{aligned}
$$

Using Eq. 13, these results yield

$$
\begin{aligned}
& \frac{\hat{K}\left(t, t^{\prime}\right)}{B^{2}\left(\lambda_{0}+\lambda_{1}\right) \operatorname{coth} \frac{\beta \hbar \omega_{0}}{2}} \\
& =\int_{-\infty}^{\infty} d \tau \frac{d G(t-\tau)}{d \tau} \frac{d G\left(t^{\prime}-\tau\right)}{d \tau} \\
& -\operatorname{Re}\left(w-v e^{2 i \phi_{B}}\right) \\
& \times \int_{-\infty}^{\infty} d \tau\left[G(t-\tau) \frac{d G\left(t^{\prime}-\tau\right)}{d \tau}+\frac{d G(t-\tau)}{d \tau} G\left(t^{\prime}-\tau\right)\right] \\
& +\left|w-v e^{2 i \phi_{B}}\right|^{2} \int_{-\infty}^{\infty} d \tau G(t-\tau) G\left(t^{\prime}-\tau\right) .
\end{aligned}
$$

From this, by noting that, from Eqs. 46, 47 and 32

$$
\left(w-v e^{2 i \phi_{B}}\right) B=-i \sqrt{2 \gamma_{1}} b_{1}^{i n} e^{i\left(\phi_{1}+\phi_{B}-\psi_{1}\right)} .
$$

and that

$$
\int_{-\infty}^{\infty} d t^{\prime} \int_{-\infty}^{\infty} d \tau G(-\tau) G\left(t^{\prime}-\tau\right)=\frac{1}{\left(\lambda_{0} \lambda_{1}\right)^{2}}
$$

one obtains, from Eq. 15 the dephasing rate

$$
\frac{1}{\tau_{\varphi}}=\lambda^{2} \frac{\lambda_{0}+\lambda_{1}}{\left(\lambda_{0} \lambda_{1}\right)^{2}} 2 \gamma_{1}\left(b_{1}^{i n}\right)^{2} \operatorname{coth} \frac{\beta \hbar \omega_{0}}{2} .
$$

Thus, $1 / \tau_{\varphi}$ is directly related to the eigenvalues $\lambda_{0}$ and $\lambda_{1}$ which characterizing the response of the detector mode to small perturbation. As was shown above, the product $\lambda_{0} \lambda_{1}$ vanishes at the points where the slope $\partial B^{2} / \partial \omega_{p}$ is infinite, leading to a diverging dephasing rate $1 / \tau_{\varphi}$. However, our model, which takes nonlinearity into account only to lowest order, breaks down near these points, where the fluctuation in $A^{\dagger} A$ becomes appreciable. To evaluate the actual dephasing rate near these points one has to take into account higher-order terms in the nonlinear expansion. This is beyond the scope of the present work, although we note that the divergence of $1 / \tau_{\varphi}$ in the present model indicates that the actual dephasing rate will be relatively large near these points. 

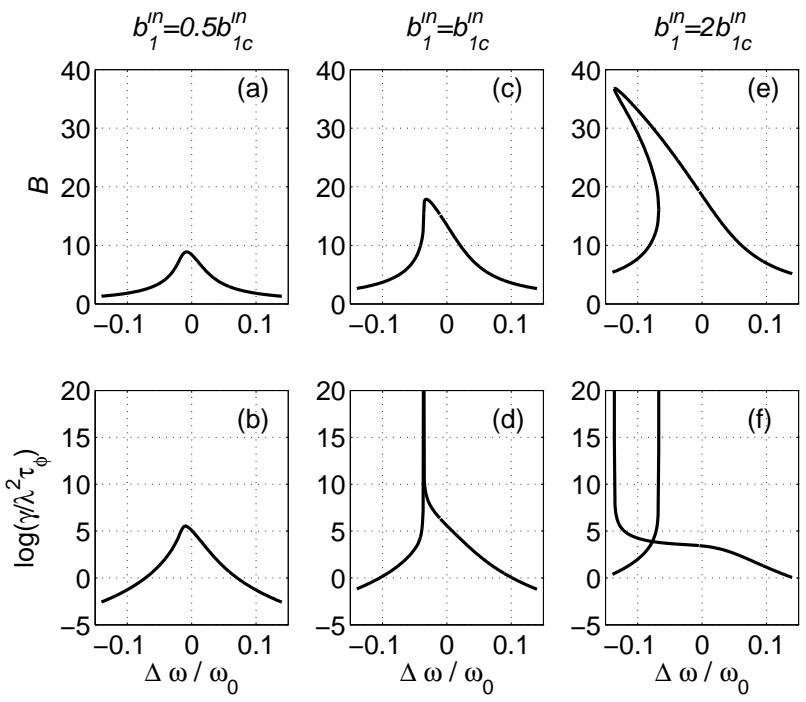

FIG. 1: Square root of average photon number in the detector mode $B$ and $\log$ of the normalized dephasing rate $\log \left(\gamma / \lambda^{2} \tau_{\varphi}\right)$, for subcritical drive $b_{1}^{i n}=0.5 b_{1 c}^{i n}$ (panels (a) and $(\mathrm{b})$ ), critical drive $b_{1}^{i n}=b_{1 c}^{i n}$ (panels (c) and (d)), and overcritical drive $b_{1}^{i n}=2 b_{1 c}^{i n}$ (panels (e) and (f)). The parameters in this example are $K=-10^{-4} \omega_{0}, \gamma_{1}=10^{-2} \omega_{0}$, $\gamma_{2}=1.1 \gamma_{1}$, and $\gamma_{3}=10^{-2} K / \sqrt{3}$.

For the linear case $K=0, \gamma_{3}=0$ the dephasing rate is given by

$$
\frac{1}{\tau_{\varphi}}=\frac{4 \lambda^{2} \gamma \gamma_{1}\left(b_{1}^{i n}\right)^{2}}{\left[\left(\omega_{0}-\omega_{p}\right)^{2}+\gamma^{2}\right]^{2}} \operatorname{coth} \frac{\beta \hbar \omega_{0}}{2} .
$$

Figure 1 shows $B$, found from solving Eq. 33 and the $\log$ of the normalized dephasing rate, $\log \left(\gamma / \lambda^{2} \tau_{\varphi}\right)$, calculated by using Eq. 70] Three cases are shown, subcritical drive $b_{1}^{\text {in }}=0.5 b_{1 c}^{\text {in }}$ (panels (a) and (b)), critical drive $b_{1}^{i n}=b_{1 c}^{i n}$ (panels (c) and (d)), and overcritical drive $b_{1}^{i n}=2 b_{1 c}^{i n}$ (panels (e) and (f)). The values chosen for this example for the parameters $K, \gamma_{1}, \gamma_{2}$, and $\gamma_{3}$ are indicated in the figure caption. As can be seen from Fig. 1 (d), the dephasing rate is an asymmetric function of frequency around the onset of bistability point, where $1 / \tau_{\varphi}$ diverges. Also, comparing the two jump points in Fig. 1 (f) indicates that the divergence in the right one (with higher frequency) is steeper.

\section{DISCUSSION}

The sensitivity of single-photon detection can be characterized by the dimensionless parameter $\xi$, defined as

$$
\xi \equiv \frac{1}{\gamma \tau_{\varphi}}
$$

Consider first the linear case $K=0, \gamma_{3}=0$, where the dephasing rate is given by Eq. 71 We assume for sim- plicity $2 \gamma_{1} \simeq \gamma$, and consider the case of low temperature $\beta \hbar \omega_{0} \gg 1$. At resonance where the dephasing rate is largest, one finds

$$
\xi=2 B^{2}\left(\frac{\lambda}{\gamma}\right)^{2} .
$$

In the present case of linear response we assume $B<$ $B_{c}$, where $B_{c}$ is given by Eq. 38 Moreover, as can be seen from Eq. A22 and A23, in many cases $K$ is of the same order as $\lambda$. Thus, assuming also the case where $\gamma_{3}<<K$, one finds that the largest possible value of $\xi$ in this regime is roughly given by

$$
\xi_{\max } \simeq \frac{\lambda}{\gamma} .
$$

The experimental data in Refs. [28] and 29], together with Eq. 38] allow one to estimate the value of $K$. Assuming, as before, that $\lambda \simeq|K|$, one finds for stripline resonators made of $\mathrm{Nb}$ that $\xi_{\max } \simeq 10^{-12}$. For resonators made of $\mathrm{NbN}$, on the other hand, the onset of nonlinear instability occurs at a much lower input power. However, the nature of the nonlinearity observed in $\mathrm{NbN}$ resonators is qualitatively different from what is expected from nonlinearity originated by the kinetic inductance effect, thus indicating that the underlying physics is different [29]. If, however, in spite of this discrepancy, we employ Eq. 38 also for the case of $\mathrm{NbN}$ resonators, we obtain $\xi_{\max } \simeq 10^{-6}$. We thus find that for both cases $\xi_{\max }$ is far too small to allow single-photon detection when the pump mode is operated in the regime of linear response. In contrast, as was mentioned above, operating the pump mode in the regime of nonlinear response may allow a significant enhancement in the sensitivity. Further study is required, however, to analyze the behavior of the system close to the points where our model yields divergence in $1 / \tau_{\varphi}$

\section{APPENDIX A: LOSSLESS TRANSMISSION LINE RESONATOR}

Consider a lossless linear transmission line with length $l$ extending along the $x$-axis [6]. Let $q(x, t)$ be the charge density per unit length and define

$$
Q(x, t)=\int_{x}^{\infty} d x^{\prime} q\left(x^{\prime}, t\right) .
$$

Thus, $q=-\partial Q / \partial x$ and the voltage across the transmission line is given by

$$
V(x, t)=-\frac{1}{C} \frac{\partial Q}{\partial x}
$$

where $C$ is the capacitance per unit length along the transmission line. The current is given by

$$
I(x, t)=\frac{\partial Q}{\partial t} .
$$


The Lagrangian of the system is given by

$$
\begin{aligned}
\mathcal{L} & =\frac{1}{2} \int_{0}^{l} d x\left[L I^{2}-C V^{2}\right] \\
& =\frac{1}{2} \int_{0}^{l} d x\left[L\left(\frac{\partial Q}{\partial t}\right)^{2}-\frac{1}{C}\left(\frac{\partial Q}{\partial x}\right)^{2}\right],
\end{aligned}
$$

where $L$ is the inductance per unit length along the transmission line. Here we assume that both $C$ and $L$ depend on $x$. Moreover, the kinetic inductance leads to a dependence of $L$ on the current, given by [1],

$$
L=L_{0}+\Delta L\left(\frac{I}{I_{c}}\right)^{2} .
$$

As a basis for expanding $Q(x, t)$ as

$$
Q(x, t)=\sum_{n} q_{n}(t) u_{n}(x),
$$

we use the solutions of the boundary value problem

$$
\frac{d}{d x}\left(\frac{1}{C} \frac{d u_{n}}{d x}\right)=-\omega_{n}^{2} L u_{n}
$$

with the boundary conditions of vanishing current $u_{n}(0)=u_{n}(l)=0$. We assume that the functions $u_{n}(x)$ are chosen to be real. Using this basis and the Strum - Liouville theorem, one obtains

$$
\mathcal{L}=\frac{1}{2} \sum_{n}\left(\dot{q}_{n}^{2}-\omega_{n}^{2} q_{n}^{2}\right)+\Delta \mathcal{L}
$$

where

$$
\begin{aligned}
\Delta \mathcal{L} & =\frac{1}{2 I_{c}^{2}} \sum_{n^{\prime}, n^{\prime \prime}, n^{\prime \prime \prime}, n^{\prime \prime \prime \prime}} \dot{q}_{n^{\prime}} \dot{q}_{n^{\prime \prime}} \dot{q}_{n^{\prime \prime \prime}} \dot{q}_{n^{\prime \prime \prime \prime}} \\
& \times \int_{0}^{l} d x \Delta L u_{n^{\prime}} u_{n^{\prime \prime}} u_{n^{\prime \prime \prime}} u_{n^{\prime \prime \prime}} .
\end{aligned}
$$

The variable canonically conjugate to $q_{n}$ is

$$
p_{n}=\frac{\partial \mathcal{L}}{\partial \dot{q}_{n}}=\dot{q}_{n}+\frac{\partial \Delta \mathcal{L}}{\partial \dot{q}_{n}} .
$$

To first order in $\Delta L$ the Hamiltonian is given by

$$
\mathcal{H}=\sum_{n} p_{n} \dot{q}_{n}-\mathcal{L}=\mathcal{H}_{0}+\mathcal{V},
$$

where

$$
\mathcal{H}_{0}=\frac{1}{2} \sum_{n}\left(p_{n}^{2}+\omega_{n}^{2} q_{n}^{2}\right)
$$

and

$$
\begin{aligned}
\mathcal{V} & =-\frac{1}{2 I_{c}^{2}} \sum_{n^{\prime}, n^{\prime \prime}, n^{\prime \prime \prime}, n^{\prime \prime \prime \prime}} p_{n^{\prime}} p_{n^{\prime \prime}} p_{n^{\prime \prime \prime}} p_{n^{\prime \prime \prime \prime}} \\
& \times \int_{0}^{l} d x \Delta L u_{n^{\prime}} u_{n^{\prime \prime}} u_{n^{\prime \prime \prime}} u_{n^{\prime \prime \prime \prime}} .
\end{aligned}
$$

Quantization is achieved by regarding the variables $\left\{q_{n}, p_{n}\right\}$ as operators satisfying the following commutation relations

$$
\left[q_{n}, p_{m}\right] \equiv q_{n} p_{m}-p_{m} q_{n}=i \hbar \delta_{n, m}
$$

and

$$
\left[q_{n}, q_{m}\right]=\left[p_{n}, p_{m}\right]=0 .
$$

In terms of the Boson annihilation and creation operators

$$
\begin{aligned}
& A_{n}=\frac{e^{i \omega_{n} t}}{\sqrt{2 \hbar}}\left(\sqrt{\omega_{n}} q_{n}+\frac{i}{\sqrt{\omega_{n}}} p_{n}\right), \\
& A_{n}^{\dagger}=\frac{e^{-i \omega_{n} t}}{\sqrt{2 \hbar}}\left(\sqrt{\omega_{n}} q_{n}-\frac{i}{\sqrt{\omega_{n}}} p_{n}\right),
\end{aligned}
$$

the Hamiltonian Eq. A11 can be expressed as

$$
\mathcal{H}_{0}=\sum_{n} \hbar \omega_{n}\left(A_{n}^{\dagger} A_{n}+\frac{1}{2}\right)
$$

The current operator is given by

$I(x, t)=\frac{\partial Q}{\partial t}=i \sum_{n} \sqrt{\frac{\hbar \omega_{n}}{2}}\left(A_{n}^{\dagger} e^{i \omega_{n} t}-A_{n} e^{-i \omega_{n} t}\right) u_{n}(x)$

and the voltage operator is given by

$$
V(x, t)=-\frac{1}{C} \sum_{n} \sqrt{\frac{\hbar}{2 \omega_{n}}}\left(A_{n}^{\dagger} e^{i \omega_{n} t}+A_{n} e^{-i \omega_{n} t}\right) \frac{d u_{n}}{d x} .
$$

The factors $p_{n^{\prime}} p_{n^{\prime \prime}} p_{n^{\prime \prime \prime}} p_{n^{\prime \prime \prime \prime}}$ generally contain terms oscillating rapidly at frequencies on the order of the frequencies in the resonator spectrum. In the RWA these terms are neglected, since their effect on the dynamics on a time scale much longer than a typical oscillation period is negligibly small, and only stationary terms remain [15]. Thus, in the expression of $\mathcal{V}$ only terms of the type $p_{n^{\prime}}^{2} p_{n^{\prime \prime}}^{2}$ contain stationary terms which are given by

$$
p_{n^{\prime}}^{2} p_{n^{\prime \prime}}^{2} \simeq \frac{\hbar \omega_{n^{\prime}}}{2} \frac{\hbar \omega_{n^{\prime \prime}}}{2}\left(1+2 A_{n^{\prime}}^{\dagger}, A_{n^{\prime}}\right)\left(1+2 A_{n^{\prime \prime}}^{\dagger} A_{n^{\prime \prime}}\right) .
$$

The constant term can be disregarded, since it only gives rise to a constant phase factor. Moreover, the terms 
$A_{n^{\prime}}^{\dagger} A_{n^{\prime}}$ and $A_{n^{\prime \prime}}^{\dagger} A_{n^{\prime \prime}}$ that give rise to frequency renormalization can be absorbed into $\mathcal{H}_{0}$. Thus, in the RWA the perturbation $\mathcal{V}$ is given by

$\mathcal{V}=\sum_{n^{\prime} \neq n^{\prime \prime}} \hbar \lambda_{n^{\prime} n^{\prime \prime}} A_{n^{\prime}}^{\dagger} A_{n^{\prime}} A_{n^{\prime \prime}}^{\dagger} A_{n^{\prime \prime}}+\sum_{n^{\prime}} \hbar K_{n^{\prime}}\left(A_{n^{\prime}}^{\dagger} A_{n^{\prime}}\right)^{2}$,

where

$$
\lambda_{n^{\prime} n^{\prime \prime}}=-\frac{3}{I_{c}^{2}} \hbar \omega_{n^{\prime}} \omega_{n^{\prime \prime}} \int_{0}^{l} d x \Delta L u_{n^{\prime}}^{2} u_{n^{\prime \prime}}^{2}
$$

and

$$
K_{n^{\prime}}=-\frac{1}{2 I_{c}^{2}} \hbar \omega_{n^{\prime}}^{2} \int_{0}^{l} d x \Delta L u_{n^{\prime}}^{4}
$$

\section{APPENDIX B: PERTURBATION THEORY}

Consider the Schrödinger equation

$$
i \frac{d}{d t}|\psi\rangle=\mathcal{K}|\psi\rangle
$$

where $\mathcal{K}=\mathcal{K}^{\dagger}$ is given by

$$
\mathcal{K}=\mathcal{K}_{0}+\lambda \mathcal{K}_{1}
$$

where $\lambda<<1$ is real. The time evolution operator $u\left(t, t_{0}\right)$ can be expanded as [30]

$$
u\left(t, t_{0}\right)=u_{0}\left(t, t_{0}\right)+\lambda u_{1}\left(t, t_{0}\right)+\lambda^{2} u_{2}\left(t, t_{0}\right)+O\left(\lambda^{3}\right),
$$

where

$$
u_{1}\left(t, t_{0}\right)=-i \int_{t_{0}}^{t} d t^{\prime} u_{0}\left(t, t^{\prime}\right) \mathcal{K}_{1}\left(t^{\prime}\right) u_{0}\left(t^{\prime}, t_{0}\right)
$$

and

$$
\begin{aligned}
& u_{2}\left(t, t_{0}\right) \\
& =-\int_{t_{0}}^{t} d t^{\prime} \int_{t_{0}}^{t^{\prime}} d t^{\prime \prime} u_{0}\left(t, t^{\prime}\right) \mathcal{K}_{1}\left(t^{\prime}\right) u_{0}\left(t^{\prime}, t^{\prime \prime}\right) \mathcal{K}_{1}\left(t^{\prime \prime}\right) u_{0}\left(t^{\prime \prime}, t_{0}\right) .
\end{aligned}
$$

[1] T. Dahm and D. J. Scalapino, J. App. Phys. 81, 2002 (1997).

[2] B. C. Sanders and G. J. Milburn, Phys. Rev. A 39, 694 (1989).

[3] V.B. Braginsky and F.Ya. Khalili, Quantum Measurement (Cambridge University Press, Cambridge, 1995).

[4] C. M. Caves, K. S. Thorne, R. W. P. Drever, V. D. Sandberg, and M. Zimmermann, Rev. Mod. Phys. 52, 341 (1980).
Using this expansion, one can calculate the operator $O(t) \equiv u_{0}^{\dagger}\left(t, t_{0}\right) u\left(t, t_{0}\right)$ to second order in $\lambda$

$O(t)$

$$
\begin{aligned}
& =1-i \lambda \int_{t_{0}}^{t} d t^{\prime} u_{0}\left(t_{0}, t^{\prime}\right) \mathcal{K}_{1}\left(t^{\prime}\right) u_{0}\left(t^{\prime}, t_{0}\right) \\
& -\lambda^{2} \int_{t_{0}}^{t} d t^{\prime} \int_{t_{0}}^{t^{\prime}} d t^{\prime \prime} u_{0}\left(t_{0}, t^{\prime}\right) \mathcal{K}_{1}\left(t^{\prime}\right) u_{0}\left(t^{\prime}, t^{\prime \prime}\right) \mathcal{K}_{1}\left(t^{\prime \prime}\right) u_{0}\left(t^{\prime \prime}, t_{0}\right)
\end{aligned}
$$

or

$$
O(t)=1-i \lambda \int_{t_{0}}^{t} d t^{\prime} \mathcal{K}_{1 H}\left(t^{\prime}\right)-\lambda^{2} \int_{t_{0}}^{t} d t^{\prime} \int_{t_{0}}^{t^{\prime}} d t^{\prime \prime} \mathcal{K}_{1 H}\left(t^{\prime}\right) \mathcal{K}_{1 H}\left(t^{\prime \prime}\right)
$$

where

$$
\mathcal{K}_{1 H}(t) \equiv u_{0}^{\dagger}\left(t, t_{0}\right) \mathcal{K}_{1}(t) u_{0}\left(t, t_{0}\right)
$$

Since $\mathcal{K}_{1}(t)$ is Hermitian, one finds to lowest order in $\lambda$

$$
\begin{aligned}
& |\langle O(t)\rangle|^{2} \\
& =1-\lambda^{2} \int_{t_{0}}^{t} d t^{\prime} \int_{t_{0}}^{t} d t^{\prime \prime}\left[\left\langle\mathcal{K}_{1}\left(t^{\prime}\right) \mathcal{K}_{1}\left(t^{\prime \prime}\right)\right\rangle-\left\langle\mathcal{K}_{1}\left(t^{\prime}\right)\right\rangle\left\langle\mathcal{K}_{1}\left(t^{\prime \prime}\right)\right\rangle\right]
\end{aligned}
$$

or

$$
|\langle O(t)\rangle|^{2}=1-\lambda^{2} \int_{t_{0}}^{t} d t^{\prime} \int_{t_{0}}^{t} d t^{\prime \prime}\left\langle\tilde{\mathcal{K}}_{1}\left(t^{\prime}\right) \tilde{\mathcal{K}}_{1}\left(t^{\prime \prime}\right)\right\rangle
$$

where

$$
\tilde{\mathcal{K}}_{1}(t)=\mathcal{K}_{1}(t)-\left\langle\mathcal{K}_{1}(t)\right\rangle
$$

\section{ACKNOWLEDGMENT}

E.B. would like to thank D. H. Santamore for fruitful discussions. This work was supported by the German Israel Foundation under grant 1-2038.1114.07, the Israel Science Foundation under grant 1380021, the Deborah Foundation, and Poznanski Foundation.
[5] N. Bohr, in P.A. Schilpp (Ed.), Albert Einstein Philosopher-Scientist, Library of Living Philosophers, Evanston, 1949, pp. 200-241.

[6] B. Yurke and J. S. Denker, Phys. Rev. A 29, 1419 (1984).

[7] B. Yurke and E. Buks, arXiv:quant-ph/0505018 (3 May 2005).

[8] C. W. Gardiner and M. J. Collett, Phys. Rev. A 31, 3761 (1985).

[9] J. Gea-Banacloche, N. Lu, L. M. Pedrotti, S. Prasad, 
M. O. Scully, and K. Wodkiewich, Phys. Rev. A 41, 369 (1990).

[10] R. Movshovich, B. Yurke, P. G. Kaminsky, A. D. Smith, A. H. Silver, R. W. Simon, and M. V. Schneider, Phys. Rev. Let. 65, 1419 (1990).

[11] S-T. Ho, X. Zhang, and M. K. Udo, J. Opt. Soc. Am. B 12, 1537 (1995).

[12] C. C. Gerrry and E. E. Hach III, Opt. Commun. 100, 211 (1993).

[13] L. Gilles, B. M. Garraway, and P. L. Knight, Phys. Rev. A 49, 2785 (1994).

[14] GX. Li, JS. Peng, and P. Zhou, Chinese Physics Letters 12, 79 (1995).

[15] D. H. Santamore, A. C. Doherty, and M. C. Cross, Phys. Rev. B 70, 144301 (2004).

[16] D. H. Santamore, H. S. Goan, G. J. Milburn, and M. L. Roukes, Phys. Rev. A 70, 052105 (2004).

[17] S. Zaitsev and E. Buks, arXiv:cond-mat/053130v1 (6 Mar 2005).

[18] A. Stern, Y. Aharonov, and Y. Imry, Phys. Rev. A 41, 3436 (1990).
[19] Y. Levinson, Europhys. Lett. 39, 299 (1997).

[20] E. Buks, R. Schuster, M. Heiblum, D. Mahalu, and V. Umansky, Physica B 251, 295 (1998).

[21] N. Tornau and A. Bach, Opt. Commun. 11, 46 (1974).

[22] G. S. Agarwal and G. P. Hildred, Opt. Comm. 58, 287 (1986).

[23] L. Gilles and P. L. Knight, Phys. Rev. A 48, 1582 (1993).

[24] H. Ezaki, J. Phys. Soc. Japan, 68, 1562 (1999).

[25] M. Kitamura and T. Tokihiro, J. Opt. B 1, 546 (1999).

[26] A. H. Nayfeh and D. T. Mook, Nonlinear Oscillations, (Wiley, 1979).

[27] L. D. Landau, Mechanics, 3rd Ed., (Pergamon, 1976).

[28] B. Abdo, E. Segev, O. Shtempluck, and E. Buks, IEEE Trans. App. Super. (to be published), arXiv:cond-mat/0501114 v2 (10 Jan 2005).

[29] B. Abdo, E. Segev, O. Shtempluck, and E. Buks, arXiv:cond-mat/0504582 (22 April 2005).

[30] Mitchel Weissbluth, Photon-Atom Interaction (Academic Press, 1989). 